\title{
Hubs and Webs in Platelet Intracellular Signalling
}

\author{
by A.N. Sveshnikova ${ }^{1-4} \#$
}

1. Center For Theoretical Problems of Physico-Chemical Pharmacology RAS, 109029, Srednyaya Kalitnikovskaya 30, Moscow, Russia

2. Dmitriy Rogachev National Medical Research Center of Pediatric Hematology, Oncology Immunology Ministry of Healthcare of Russian Federation, 117997, Samory Machela 1, Moscow Russia

3. Lomonosov Moscow State University, Physics Faculty 119991, Leninskiye Gory 1-2, Moscow, Russia

4. Department of Normal Physiology, Sechenov First Moscow State Medical University, 8/2 Trubetskaya St., Moscow, 119991 , Russia

\# Correspondence: a.sveshnikova@physics.msu.ru

Received: 30.09.2021

Accepted: $\mathbf{3 0 . 0 9 . 2 0 2 1}$

Published: 30.09 .2021

DOI: 10.52455/sbpr.01.202103014

\section{Editorial Letter}

In this issue of Systems Biology and Physiology Reports, A.A. Martyanov and M.A. Panteleev suggested a review on platelet intracellular signalling network, which is a second part in the discussion on the molecular relationships between platelet activation and responses (1). The review contains seven thousands words and two hundred references and yet it is not complete, as there are still unclear parts in platelet signaling, especially in its inhibition (2-4). In an effort to comprehend the platelet activation pattern, I drew a signalling scheme based on the review and data from other authors $(2,3)$ (Fig. 1).

One could see from the Figure that there is no clear isolated "activation sequence" or "activation cascade" from any receptor. Platelet signalling pathways tend to tightly interact with each other and cross-link on several levels. For example, both pathways from PARs and GPVI activate PLCs and PI3Ks, and cytosolic calcium regulates both PLCs. Cytosolic calcium was suggested to be the main regulator of platelet activation (5) and, indeed, it regulates most of the platelet functional responses (1). Therefore, cytosolic calcium could be called a "Hub", which summarizes information on platelet activation (6) However, there are several other candidates for signalling hubs: all platelet GPCRs use the same Gßy, which is crucial for platelet integrin activation (7), all platelet glycoprotein receptors activate SFK and Syk kinases, and all platelet receptors activate PI3Ks of different types. With addition of cross-regulation, these interactions form a spider-web, which is hard to analyze (Fig. 1).

While linear description could be useful for memorizing platelet signalling, it is misleading for its comprehension. The realistic description of platelet signal transduction is that of a network with several levels of intersection, with bifurcations and junctions. Any attempt to understand the mechanism of information transmission in the signalling network should consider the system as a whole, in the entirety of its architecture.

It is not easy; moreover, it is not likely that this is something to be done mentally. Non-linear interactions, oscillations and spiking, frequency and amplitude coding/decoding could be only analyzed with the help of mathematical and computer modelling $(6,8-10)$. I believe that this line of approach is going to be incrementally important in deciphering the meaning behind the platelet signalling design, and will hopefully lead to the development of efficient tools for diagnostics and treatment based on this understanding.

\section{References}

1. Sveshnikova A, Stepanyan M, Panteleev M, Sveshnikova A, Stepanyan $M$, Panteleev $M$. Platelet functional responses and signalling: the molecular relationship. Part 1: responses. Systems Biology and Physiology Reports. 2021 Mar;1(1):20.

2. Makhoul S, Walter E, Pagel O, Walter U, Sickmann A, Gambaryan S, et al. Effects of the NO/soluble guanylate cyclase/cGMP system on the functions of human platelets. Nitric Oxide. 2018 Jun 1;76:71-80.

3. Vögtle T, Sharma S, Mori J, Nagy Z, Semeniak D, Scandola C, et al. Heparan sulfates are critical regulators of the inhibitory megakaryocyteplatelet receptor G6b-B. Bjorkman PJ, Cole PA, editors. eLife. 2019 Aug 22;8:e46840.

4. Lin C, Koval A, Tishchenko S, Gabdulkhakov A, Tin U, Solis GP, et al. Double Suppression of the Ga Protein Activity by RGS Proteins. Molecular Cell. 2014;53(4):663-71.

5. Versteeg $\mathrm{HH}$, Heemskerk JWM, Levi M, Reitsma PH. New fundamentals in hemostasis. Physiol Rev. 2013;93(1):327-58.

6. Sveshnikova AN, Balatskiy AV, Demianova AS, Shepelyuk TO, Shakhidzhanov SS, Balatskaya MN, et al. Systems biology insights into the meaning of the platelet's dual-receptor thrombin signaling. Journal of Thrombosis and Haemostasis. 2016;14(10):2045-57.

7. Stefanini L, Bergmeier W. RAP GTPases and platelet integrin signaling Platelets. 2019;30(1):41-7.

8. Dunster JL, Panteleev MA, Gibbins JM, Sveshnikova AN. Mathematical Techniques for Understanding Platelet Regulation and the Development of New Pharmacological Approaches. In: Gibbins JM, Mahaut-Smith M, editors. Platelets and Megakaryocytes : Volume 4, Advanced Protocols and Perspectives [Internet]. New York, NY: Springer; 2018 [cited 2020 Jul 28]. p. 255-79. (Methods in Molecular Biology). Available from: https://doi.org/10.1007/978-1-4939-8585-2_15

9. Purvis JE, Chatterjee MS, Brass LF, Diamond SL. A molecular signaling model of platelet phosphoinositide and calcium regulation during homeostasis and P2Y1 activation. Blood. 2008;112(1528-0020 (Electronic)):4069-79.

10. Martyanov AA, Balabin FA, Dunster JL, Panteleev MA, Gibbins JM, Sveshnikova AN. Control of Platelet CLEC-2-Mediated Activation by Receptor Clustering and Tyrosine Kinase Signaling. Biophysical Journal. 2020 Apr;S0006349520303465. 


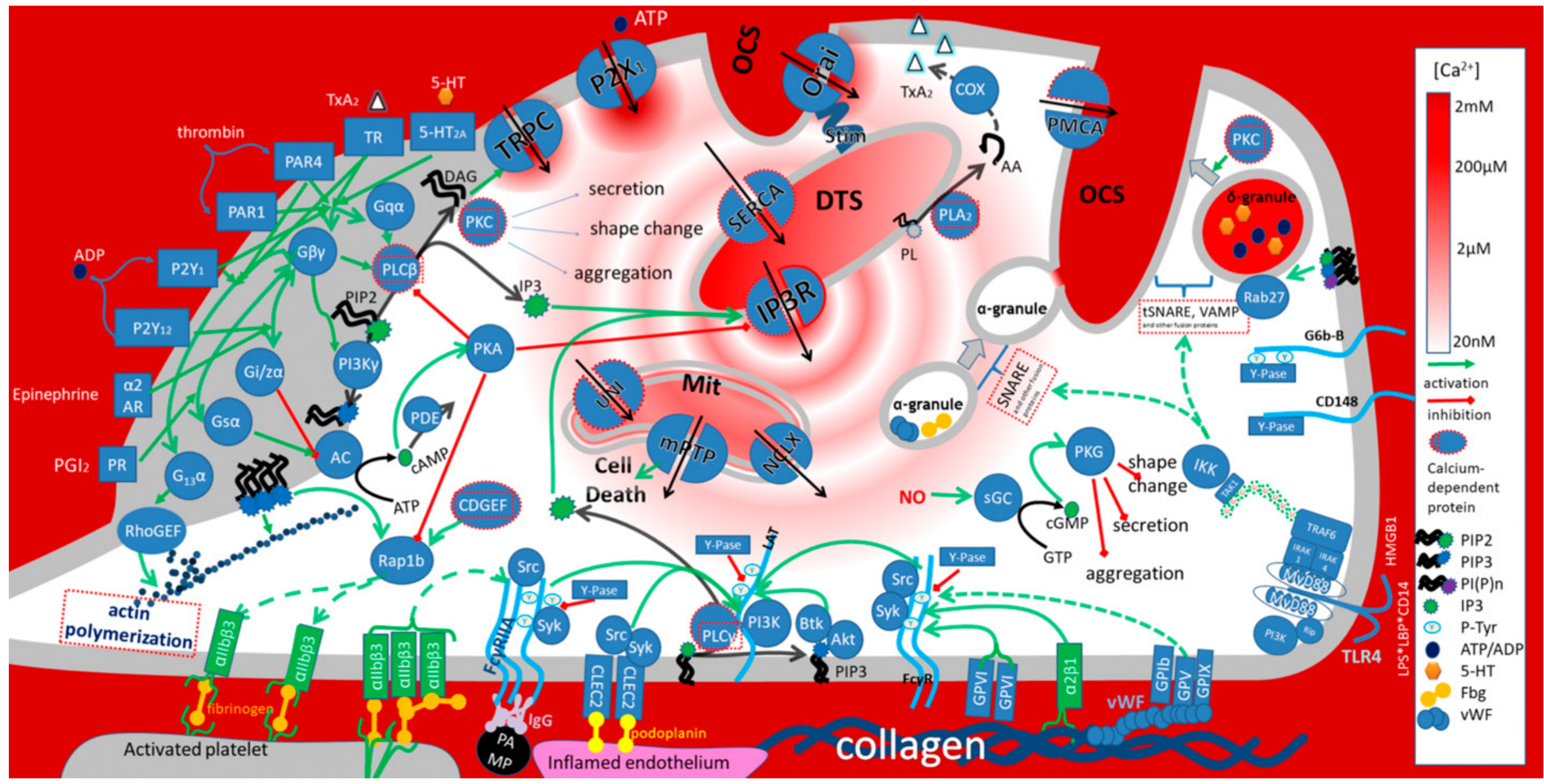

Figure. Scheme of platelet intracellular signalling with focus on cytosolic calcium as a "Hub". Cytosolic calcium concentration is given in the shades of red. Almost all types of platelet receptor agonists lead to activation of phospholipase C (PLC), followed by calcium release from intracellular stores (DTS). Calcium concentration is rapidly reduced by calcium-dependent ATPases (SERCA and PMCA). Also, it could be reduced by binding with some buffering proteins, including its effector-proteins (indicated with red dots). Platelet mitochondria also can function as a calcium buffer and, simultaneously, be regulated by calcium concentration. Direct activation is shown by solid green arrows, direct inhibition - by solid red arrows. Indirect interactions are shown by dashed lines. Abbreviations. AC - adenylate cyclase, a2AAR - a2A-adrenergic receptor, CDGEF - CalDAGGEFI, COX - cyclooxygenase, DAG - diacylglycerol, DTS - dense tubular system, Fbg - fibrinogen, IP3R - receptor for inositol-1,4,5-trisphosphate (IP3), Mit - a mitochondrion, mPTP - mitochondrial permeability transition pore, NCLX - mitochondrial sodium/calcium exchanger, OCS - open canalicular system, P2Y - purinergic receptor, PAR - protease-activated receptor, PIP2 phosphoinositol-4,5-bisphosphate, PIP3 - phosphoinositol-3,4,5-trisphosphate, PI(P)n - phosphoinositides, PKA - protein kinase A, PKG - protein kinase G, PKC - protein kinase C, PL - phospholipid, PLA2 - phospholipase A2, PMCA - plasma membrane calcium ATPase, PR - PGI2 receptor, P-Tyr - phosphorylated tyrosine residue, SERCA - sarcoplasmic/endoplasmic reticulum calcium ATPase, SGC - soluble guanylate cyclase, TR - thromboxane A2 (TxA2) receptor, TRPC - transient receptor potential channel, UNI - mitochondrial uniporter, vWF - von Willebrand Factor, Y-Pase - tyrosine phosphatase. 